\title{
Energy Detection based Spectrum Sensing with Constraint Region in Cognitive LTE Systems
}

\author{
Nan Wang, Yue Gao, Fengyi Yang, Qi Bi, Weiliang Xie, and Clive Parini
}

\begin{abstract}
Cognitive LTE system, such as LTE-U, has received tremendous interest from almost all research disciplines in wireless communications. In Cognitive LTE systems, spectrum sensing is the key enabling technology to realize dynamic spectrum access. In this paper, an error decision probability is proposed to solve the trade-off problem between detection and false alarm probability with the constraint region requirement. The closedform expression for the error decision probability, satisfied SNR value, number of samples and primary users' spectrum utilization ratio are derived for in both fixed and adaptive threshold setting algorithms. By implementing both Welch and wavelet based energy detectors, the adaptive threshold setting algorithm demonstrates a more reliable and robust sensing result for both primary users (PUs) and secondary users (SUs) in comparison with the conventional fixed one. Furthermore, the wavelet de-noising method is applied to improve the sensing performance when there are insufficient number of samples.
\end{abstract}

Index Terms-Spectrum sensing, energy detection, adaptive threshold, wavelet denoising

\section{INTRODUCTION}

C OGNITIVE radio (CR) is being viewed as a new intelligent wireless communication technology to improve efficiency of a fixed spectrum assignment policy [1], [2]. In the future the fifth generation (5G) networks, there has been an increasing interest [3] of enhancing the mainstream cellular technology LTE with CR for accessing a much wider range of spectrum bands to improve the data throughput. Long-term evolution in unlicensed spectrum (LTE-U) can be considered as a kind of cognitive LTE system for expanding cellular network capacity without additional spectrum cost. For dynamically accessing wider range of spectrum bands in a friendly manner, the cognitive LTE system should have the capability of discovering the possible coexisting heterogeneous wireless systems in different bands and be able to avoid the mutual interference with them. However, this new requirement is beyond LTE's conventional capability of coexisting with neighboring cells based on the measurement function of User Equipment (UE) [4]. Thanks to the spectrum sensing techniques of CR, the cognitive LTE system could monitor the dynamic spectrum environment to avoid the mutual interference with possible coexisting heterogeneous wireless systems in different bands.

The spectrum sensing is one of the most challenging tasks in the future cognitive LTE system as it requires high accuracy

Nan Wang, Fengyi Yang, Qi Bi and Weiliang Xie are with China Telecom Technology Innovation Center, Beijing, 102209, China (email:\{wangnan, yangfy, qibi, xiewl\}@ctbri.com.cn)

Y. Gao and Clive Parini are with Queen Mary University of London, School of Electronic Engineering and Computer Science, London E1 4NS, United Kingdom (email:\{yue.gao, c.g.parini\}@qmul.ac.uk) and low complexity for dynamic spectrum access [5] [6]. The spectrum sensing performance metric is usually measured as a trade-off between selectivity and sensitivity, and can be quantified by the levels of detection and false alarm probability. The higher the detection probability, the better primary users (PUs) can be protected. The lower the false alarm probability, the more chances a channel can be utilized by secondary users (SUs). A cooperative spectrum sensing with joint energy and correlation detection has been proposed to decrease the probability of false alarm and miss-detection [7]. In [8], a new detection based on four-level hypothesis for blind spectrum sensing has been proposed to decrease the probability of false alarm while avoiding interfering to the primary system. A detection probability of $90 \%$ and a false alarm probability of $10 \%$ have been set as the target requirements in the IEEE 802.22 standard for all the sensing algorithms [9], [10].

The energy detection, also known as radiometry or periodogram, is the most common way of spectrum sensing because of its low computation and implementation complexities [11]. The sensing performance of energy detection depends greatly on the setting of a detection threshold. Most conventional energy detection methods adopt a fixed decision threshold to distinguish PU signals from the noise. For example, a predefined experimental threshold was set in [12], [13] by measuring the noise power. However, it is difficult to guarantee the detection and false alarm probability with the fixed threshold setting method, especially when the noise power fluctuates [14], [15]. Unlike the fixed-threshold-based sensing algorithm, an adaptive spectrum sensing algorithm was proposed in our previous research [16] to make the SU can dynamically adjust its energy threshold according to the Signalto-Noise ratio (SNR) and varying PUs' spectrum utilizations. Besides, a hybrid framework combining compressive spectrum sensing with geo-location database was proposed in [17]. By implementing previously developed power control based geolocation database and adaptive spectrum sensing algorithm, a database-augmented sensing algorithm was proposed in [18] for a secondary access of the TV white space spectrum.

However, both fixed and adaptive threshold based energy detection should also satisfy the IEEE 802.22 constraint region requirement on $P_{d} \geq 90 \%$ and $P_{f} \leq 10 \%$ [9]. A number of research work on the constraint region problem has been conducted. In [19], the proposed cooperative sensing techniques could achieve above $90 \%$ detection probability at $S N R=-7 d B$ with observation window of 50bits and $10 \%$ false alarm rate. For $10 \%$ false alarm probability, $90 \%$ detection probability of BPSK signals with SNR of $-8 \mathrm{~dB}$ or greater was achieved the cyclostationary spectrum detection in [20]. Both [19] and [20] couldn't detect signal at low SNR as required by the Federal Communications Commission (FCC) in US [21] and 
the Office of Communications (Ofcom) in UK [22]. In [23], the proposed cyclostationary spectrum density (CSD) estimation could achieve greater than $90 \%$ detection probability on BPSK signals with SNR of $-18 \mathrm{~dB}$, when the probability of false alarm is less than $10 \%$. In [24], the proposed energy detection scheme for multi-carrier systems could reach $90 \%$ in probability of detection and $10 \%$ in probability of false alarm for the SNRs as low as $-21 \mathrm{~dB}$. Although the achieved sensing SNR in both [23] and [24] could maintain a relative low level, the effect of varying PUs' spectrum utilizations on the sensing performance was not taken into account. Therefore, a closed-form expression between PUs' spectrum utilization ratio and the threshold is derived by considering the constraint region required by the IEEE 802.22 standard in this paper. The impacts of different PUs' utilizations on the sensing performance are also quantified. Moreover, both conventional fixed and proposed adaptive threshold setting algorithms are implemented with Welch and wavelet based energy detectors for sensing OFDM signals at SNR values ranging from $-25 \mathrm{~dB}$ to $0 \mathrm{~dB}$. The sensing performance is further improved by the wavelet de-noising method.

The remainder of this paper is organized as follows: a generic CR system model is provided in Section II. The spectrum sensing algorithms of both Welch and wavelet based energy detector are described in Section III. The fixed threshold setting algorithm considering the constraint region requirement is investigated in Section IV. A modified adaptive threshold setting algorithm is presented in Section V with considering the constraint region requirement. Simulations of both proposed and conventional algorithms are compared and analyzed in Section VI by implementing with Welch and wavelet based energy detector. The conclusions are drawn in Section VII.

\section{SySTEM MODEL}

In this paper, an LTE-A system with cognitive radio enhancement is being developed based on our previous study [25]. Cognitive User Equipments (UE) are communicating with its associated LTE eNodeB base station in Frequency-Division Duplexing (FDD) manner and tries detect the possible emergence of coexisting LTE signals. Therefore, the PU represents the LTE signal, and the SU represents the cognitive UE. The energy detector at SUs is used to detect the presence of PU signals. It firstly measures the power of the input PU signals over a time interval $T$, then the received power is compared to a predefined fixed threshold to decide whether the frequency band is occupied or not. The sensing decision can be formulated into a binary hypothesis problem by

$$
\begin{aligned}
& H_{0}: y(n)=w(n) \quad(\text { signal absent }) \\
& H_{1}: y(n)=h(n) s(n)+w(n)(\text { signal present })
\end{aligned}
$$

where $H_{0}$ and $H_{1}$ denote the hypothesis PU absent and PU present, respectively. After bandpass filtering over a bandwidth $W$, the received signal is denoted as $y(n)(n=0,1, \ldots, N-1) . w(n)$ represents the additive white Gaussian noise, assumed to be independent and identically distributed (iid) with zero mean and variance of $\sigma_{n}^{2} . s(n)$ is the PU signal, also assumed to be an iid random process with zero mean and variance of $\sigma_{s}^{2}$. $h(n)$ is the channel gain with an average value of $\sigma_{h}^{2}$. With the signal and noise variance, the average SNR can be defined as $S N R=\sigma_{h}^{2} \cdot \sigma_{s}^{2} / \sigma_{n}^{2}[26]$.

The performance metric of spectrum sensing can be measured by the detection probability $P_{d}$ and the false alarm probability $P_{f}$. When the number of samples $N$ is large enough, $P_{d}$ can be derived by [27], [28]

$$
P_{d}=P\left(Y>\lambda \mid H_{1}\right)=Q\left(\frac{\lambda-\left(\sigma_{n}^{2}+\sigma_{h}^{2} \cdot \sigma_{s}^{2}\right)}{\left(\sigma_{n}^{2}+\sigma_{h}^{2} \cdot \sigma_{s}^{2}\right) / \sqrt{N / 2}}\right)
$$

and $P_{f}$ can be given as

$$
P_{f}=P\left(Y>\lambda \mid H_{0}\right)=Q\left(\frac{\lambda-\sigma_{n}^{2}}{\sigma_{n}^{2} / \sqrt{N / 2}}\right)
$$

where $Y$ stands for the average energy. Without loss of generality, we assume $\sigma_{h}^{2}=1$, and the average SNR is $\bar{\gamma}=\sigma_{s}^{2} / \sigma_{n}^{2}$. It can be seen that both $P_{d}$ and $P_{f}$ are mainly dependent on the threshold $\lambda$, if the signal variance $\sigma_{s}^{2}$, the noise variance $\sigma_{n}^{2}$ and the number of samples $N$ are known. Therefore, the decision threshold can be derived for a target $P_{d}$ or $P_{f}$. Under hypothesis $H_{1}$, the threshold $\lambda_{P_{d}}$ can be set for a constant detection rate (CDR) as [29]

$$
\lambda_{P_{d}}=\left(\sigma_{n}^{2}+\sigma_{s}^{2}\right)\left(1+\frac{Q^{-1}\left(P_{d}\right)}{\sqrt{N / 2}}\right)
$$

Similarly, under hypothesis $H_{0}$, the threshold $\lambda_{P_{f}}$ can be set for a constant false alarm rate (CFAR) as

$$
\lambda_{P_{f}}=\sigma_{n}^{2}\left(1+\frac{Q^{-1}\left(P_{f}\right)}{\sqrt{N / 2}}\right)
$$

It has been shown in (4) and (5) that the threshold derivation results are similar for both CDR and CFAR. The threshold based on CFAR is commonly applied in conventional energy detection algorithms.

\section{Spectrum Sensing Algorithms}

\section{A. Welch's Energy Detection}

Welch's algorithm is a modified periodogram. The principle of the Welch algorithm is to divide the data sequence into segments in order to reduce the large fluctuations of the periodogram [13]. For instance, a signal $s(n)$ is segmented into $M$ segments in the time domain with length $L$ for each segment. $L$ is the number of frequency bins to be averaged around the zero frequency. Therefore, an input signal $s(n)$ can be defined as a matrix with $L \times M$ elements

$$
s(m, l)=s(l+(m-1) \cdot(L-1))
$$

where $m=1, \ldots M$ and $l=1, \ldots L$. After partitioning the input signal $s(n)$ into $M$ segments, FFT is first applied to each segment, and averaging is then performed over the squared outputs of the FFT. At this point, the average signal energy of $s(n)$ in the frequency domain can be presented by:

$$
P(l)=\frac{1}{M} \sum_{m=1}^{M}[|F F T(s(m, l))|]^{2}
$$


The signal energy $P(l)$ is averaged over $L$ samples in the frequency domain. The average energy over the entire frequency band, $Y$, is obtained:

$$
Y=\frac{1}{L} \sum_{l=-L / 2}^{L / 2} P(l) \gtrless \lambda
$$

This average energy $Y$ is then compared with the threshold $\lambda$ obtained from either (4) or (5) to determine whether the primary signal is present or not.

\section{B. Discrete Wavelet Packet Transform based Energy Detection}

Discrete wavelet transform (DWT) is designed from the multi-resolution analysis, decomposes the given signal space into an approximate space $\mathrm{V}$ and detail space $\mathrm{W}$ as [30]

$$
V_{j}=W_{j} \oplus V_{j-1}=W_{j} \oplus W_{j-1} \oplus V_{j-1}
$$

where $W_{j}$ is the orthogonal complement of $V_{j}$ in $V_{j+1}$ and $\oplus$ represents the orthogonal sum of the subspace. Two spaces $V_{j}$ and $W_{j}$ are constructed by orthogonal scaling functions $\phi_{j, k}$ and orthogonal wavelet functions $\psi_{j, k}$, respectively. The scaling function $\phi_{j, k}$ and the wavelet function $\psi_{j, k}$ are obtained as

$$
\begin{aligned}
& \phi_{j, k}(t)=2^{j / 2} \cdot \phi\left(2^{j} t-k\right)=\sum_{l} h_{l-2 k} \cdot \phi_{j+1, k(t)} \\
& \psi_{j, k}(t)=2^{j / 2} \cdot \psi\left(2^{j} t-k\right)=\sum_{l} g_{l-2 k} \cdot \psi_{j+1, k(t)}
\end{aligned}
$$

with low pass filter $h_{l-2 k}=\left\langle\phi_{j, k}, \phi_{j+1, l}\right\rangle$ and high-pass filter $g_{l-2 k}=\left\langle\psi_{j, k}, \psi_{j+1, l}\right\rangle$. \langle\rangle means inner product. Using these functions, the DWT of a given signal $s(t)$ provides scaling coefficients and wavelet coefficients. The scaling coefficient at the $j^{\text {th }}$ level and $k^{\text {th }}$ time is computed by:

$$
c_{j, k}=\left\langle s, \phi_{j, k}\right\rangle=\sum_{l} h_{l-2 k}^{*}\left\langle s, \phi_{j+1, l}\right\rangle=\sum_{l} h_{l-2 k}^{*} c_{j+1, l}
$$

The wavelet coefficient at the $j^{\text {th }}$ level and $k^{\text {th }}$ time is

$$
d_{j, k}=\left\langle s, \psi_{j, k}\right\rangle=\sum_{l} g_{l-2 k}^{*}\left\langle s, \psi_{j+1, l}\right\rangle=\sum_{l} g_{l-2 k}^{*} c_{j+1, l}
$$

Discrete wavelet packet transform (DWPT) based energy detection can be thought of as a modified DWT method, in which DWPT decomposes not only the approximation space but also the detail space [30]. In other words, DWPT can separate the frequency band uniformly. In order to improve the time-frequency resolution, the DWPT decomposition algorithm is adopted to divide the received signal in this paper.

With DWPT, a signal can be represented as

$$
s(t)=\sum_{j \geq j_{0}} \sum_{k}\left(c_{j, k} \varphi_{j, k}(t)+d_{j, k} \psi_{j, k}(t)\right)
$$

where $c_{j, k}$ and $d_{j, k}$ are the scaling and wavelet coefficients, respectively. $\varphi_{j, k}(t)$ and $\psi_{j, k}(t)$ are the wavelet bases to describe the approximation and detailed space of a signal respectively. $j_{0}$ denotes the computation starts from the first level wavelet coefficient. The energy of signal $s(t)$ can be measured with the two coefficients as

$$
\begin{aligned}
& E=\frac{1}{T} \int_{0}^{T}\left[\sum_{j \geq j_{0}} \sum_{k} c_{j, k} \varphi_{j, k}(t)+d_{j, k} \psi_{j, k}(t)\right]^{2} d t \\
& =\frac{1}{T} \sum_{j \geq j_{0}} \sum_{k}\left(c_{j, k}{ }^{2}+d_{j, k}{ }^{2}\right) \gtrless \lambda
\end{aligned}
$$

The obtained energy $E$ is then compared to the threshold $\lambda$ obtained from either (4) or (5) to determine whether the primary signal is present or not.

\section{The Proposed Wavelet de-noising}

After a wavelet transform, there are normally more wavelet coefficients of noise than those of signals. However, the amplitudes of signal's wavelet coefficients are much higher than that of the noise. The wavelet de-noising method was proposed in [31] to remove the noise and improve the SNR. The wavelet de-noising process is described as follows in Algorithm 1.

\section{Algorithm 1 Wavelet De-noising Process \\ Initiazation:}

1: The received signal $s(n)$ 's wavelet packet coefficients $\left(w_{j, k}\right)$ are first obtained after performing $n$ level DWPT.

2: Compare the wavelet coefficients in the wavelet packet tree with a threshold $(T)$ to generate a new wavelet packet coefficients $\left(w^{*}{ }_{j, k}\right)$. Here, the VisuShrink threshold is used [32].

$$
T=\sigma_{n} \sqrt{2 \ln (N \log 2(N))} ;
$$

where $\sigma_{n}$ is the noise standard derivation and $\mathrm{N}$ is the length of the received primary signal. The adaptive threshold value makes the value of $\left|w_{j, k}-w^{*}{ }_{j, k}\right|$ as small as possible.

\section{Decision:}

The revised wavelet packets coefficients $\left(w^{*}{ }_{j, k}\right)$ are used to reconstruct the estimated original signal.

For the fixed threshold based sensing algorithms, the energy obtained from both (8) and (14) is compared to either the CDR threshold in (4) or the CFAR threshold in (5). However, this conventional fixed threshold based sensing process faces one problem that it only considers one aspect every time in the favour of either PUs or SUs. If the CR network is designed to guarantee PUs' safe use of the spectrum, the CDR method should be used and the target detection probability $P_{d}$ should be set as high as possible. The higher the detection probability, the better the PUs can be protected. If the CR network is designed to guarantee the spectrum efficiency of the SUs, the CFAR method should be implemented and the target false alarm probability $P_{f}$ should be set as small as possible. The lower the false alarm probability, the more chances a channel can be utilized by SUs. In order to maximize the benefit for both PUs and SUs, the trade-off problem between $P_{d}$ and $P_{f}$ is investigated thoroughly. Furthermore, the constraint region problem should be considered to meet the IEEE 802.22 requirement on $90 \%$ probability and $10 \%$ false alarm probability. 


\section{The FiXed Threshold Setting Algorithm with CONSTRAINT REgION}

In this paper, the trade-off problem between $P_{d}$ and $P_{f}$ is formulated to an equivalent form of minimizing the error decision probability $P_{e}$ as a function of PUs' spectrum utilization ratio $\alpha(0<\alpha<1)$ and the threshold $\lambda$ as

$$
\begin{gathered}
\operatorname{minimize}\left(P_{e}(\lambda)\right)=\operatorname{minimize}\left\{(1-\alpha) P_{f}+\alpha\left(1-P_{d}\right)\right\} \\
\text { subject to: } P_{f} \leq 10 \% \\
P_{d} \geq 90 \%
\end{gathered}
$$

where $(1-\alpha)$ stands for the probability that the PU channel is vacant and full of noise, $\left(1-P_{d}\right)$ represents the missed detection probability that indicates PUs being absent while actually present. Therefore, the weighted error decision probability for PUs being present is $\alpha\left(1-P_{d}\right)$ with PUs' spectrum utilization $\alpha$. Similarly, $(1-\alpha) P_{f}$ is the weighted error decision probability for PUs being absent. According to the IEEE 802.22 standard, the detection and false alarm probability should keep a sensing level of $90 \%$ and $10 \%$, respectively. Thus, the total error decision probability $P_{e}$ should be minimized as much as possible to maximize the benefit for both PUs and SUs subject to the two conditions $P_{d} \geq 90 \%$ and $P_{f} \leq 10 \%$.

Substitute $P_{d}$ in (2) and $P_{f}$ in (3) into (16), the optimization problem can be mathematically formulated as

$$
\begin{aligned}
& P_{e}(\lambda)=(1-\alpha) P_{f}+\alpha\left(1-P_{d}\right) \\
& =(1-\alpha) Q\left(\frac{\lambda-\sigma_{n}^{2}}{\sigma_{n}^{2} / \sqrt{N / 2}}\right)+\alpha\left[1-Q\left(\frac{\lambda-\left(\sigma_{n}^{2}+\sigma_{s}^{2}\right)}{\left(\sigma_{n}^{2}+\sigma_{s}^{2}\right) / \sqrt{N / 2}}\right)\right] \\
& =\frac{1-\alpha}{\sqrt{\pi}} \int_{\frac{a}{\sqrt{2}}}^{\infty} e^{-z^{2}} d z-\frac{\alpha}{\sqrt{\pi}} \int_{\frac{b}{\sqrt{2}}}^{\infty} e^{-z^{2}} d z+\alpha
\end{aligned}
$$

where $a=\frac{\left(\lambda-\sigma_{n}^{2}\right)}{\sigma_{n}^{2}} \cdot \sqrt{N / 2}$ and $b=\frac{\lambda-\left(\sigma_{n}^{2}+\sigma_{s}^{2}\right)}{\sigma_{n}^{2}+\sigma_{s}^{2}} \cdot \sqrt{N / 2}$.

Based on equation (2), (3), $Q(1.28)=0.1$ and $Q(-1.28)=$ 0.9 , the trade-off optimization problem considering the constraint region can be mathematically formulated as

$$
\begin{gathered}
\text { min: } f(\lambda)=\frac{1-\alpha}{\sqrt{\pi}} \int_{\frac{a}{\sqrt{2}}}^{\infty} e^{-z^{2}} d z-\frac{\alpha}{\sqrt{\pi}} \int_{\frac{b}{\sqrt{2}}}^{\infty} e^{-z^{2}} d z+\alpha \\
\text { subject to: } g_{1}(\lambda) \leq 0 \\
g_{2}(\lambda) \leq 0
\end{gathered}
$$

where $g_{1}(\lambda)=\lambda+\frac{1.28\left(\sigma_{n}^{2}+\sigma_{s}^{2}\right)}{\sqrt{N / 2}}-\sigma_{n}^{2}-\sigma_{s}^{2}, g_{2}(\lambda)=-\lambda+$ $\frac{1.28 \sigma_{n}^{2}}{\sqrt{N / 2}}+\sigma_{n}^{2}$ are the inequality constraint equations.

For the conventional fixed threshold based sensing algorithm,either CFAR or CDR method is applied. Here, the CFAR method is used as an example just as the majority of research did in the energy detection field. Substitute (5) into $g_{2}(\lambda)$, we have $Q^{-1}\left(P_{f}\right) \geq 1.28$, which means $P_{f} \leq 10 \%$. Substitute (5) into $g_{1}(\lambda)$, we have

$$
\sigma_{s}^{2} \geq \frac{1.28\left(\sigma_{n}^{2}+\sigma_{s}^{2}\right)+\sigma_{n}^{2}\left(Q^{-1}\left(P_{f}\right)\right)}{\sqrt{N / 2}}
$$

(19) can be further simplified as

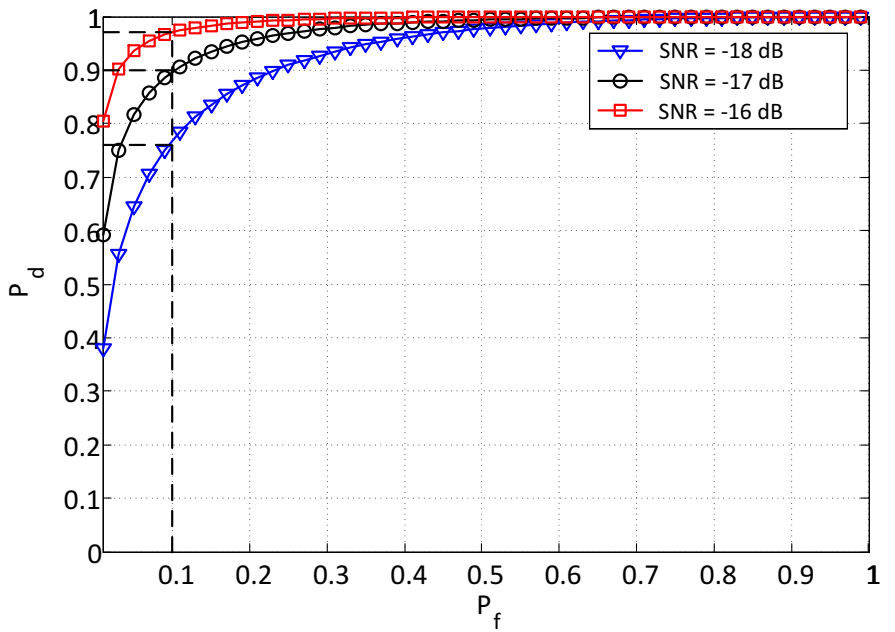

Fig. 1: $P_{d}$ versus $P_{f}$ for different SNR with $N=16384$

$$
S N R=\frac{\sigma_{s}^{2}}{\sigma_{n}^{2}} \geq \frac{\sqrt{N / 2}-1.28}{Q^{-1}\left(P_{f}\right)+1.28}
$$

Therefore (18) can be further formulated as

$$
\begin{gathered}
\text { min }: f(\lambda)=\frac{1-\alpha}{\sqrt{\pi}} \int_{\frac{a}{\sqrt{2}}}^{\infty} e^{-z^{2}} d z-\frac{\alpha}{\sqrt{\pi}} \int_{\frac{b}{\sqrt{2}}}^{\infty} e^{-z^{2}} d z+\alpha \\
\text { subject to: } P_{f} \leq 10 \% \\
S N R \geq \frac{Q^{-1}\left(P_{f}\right)+1.28}{\sqrt{N / 2}-1.28}
\end{gathered}
$$

The solutions for (21) can be obtained by discussing all the possible cases of SNR and the number of samples $N$. For example, if $P_{f}=0.1, N=16384$, the corresponding SNR should be greater than $-17 \mathrm{~dB}$ to make (21) hold and generate the minimum error decision probability at $S N R=-17 d B$. In order to verify our derivation, Fig. 1 is obtained by substituting variable SNR values into (21). It can be found from Fig. 1 that the detection probability $P_{d}$ will be higher than $90 \%$ if SNR is greater than $-17 \mathrm{~dB}$, otherwise, a lower detection probability $P_{d}$ will be obtained.

Similarly, the required number of samples $N$ satisfying the constraint condition could also be found for a given SNR value as shown in Fig. 2. It is shown in Fig. 2 that the minimum number of samples $N$ to satisfy the $90 \% P_{d}$ and $10 \% P_{f}$ constraint region should be at least 16384. The larger the number of samples $N$, the easier the constraint region problem could be satisfied. These research results a theoretical guidance when designing the energy detector to satisfy the constraint region requirement in reality.

\section{The Adaptive Threshold Setting Algorithm WITH CONSTRAINT REGION}

Besides the conventional fixed threshold setting algorithm, the constraint region problem is further extended to our previously proposed adaptive threshold setting algorithm [16] in this section. According to the method of Lagrange multipliers and Karush-Kuhn-Tucker (KKT) conditions [33], the objection 


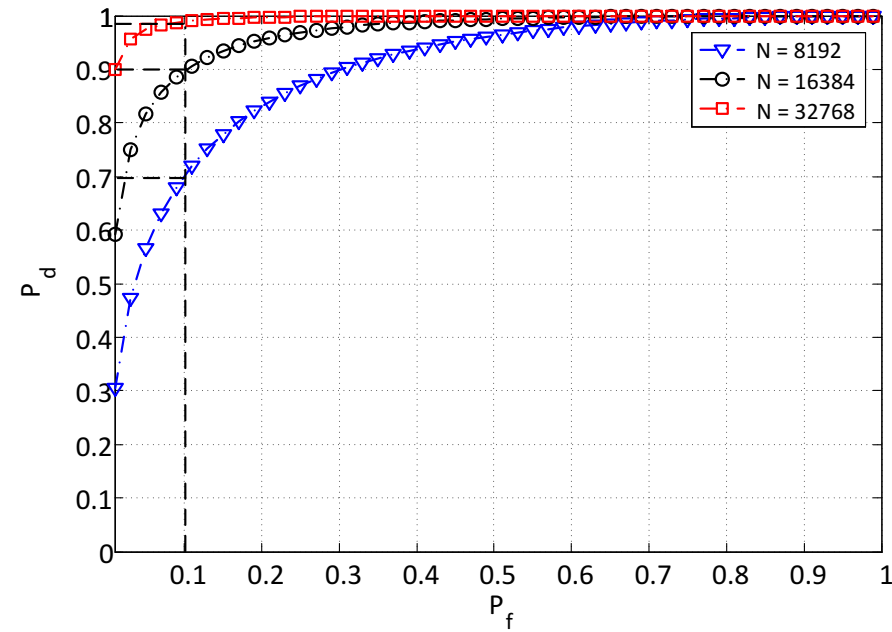

Fig. 2: $P_{d}$ versus $P_{f}$ for different number of samples $N$ at $S N R=-17 d B$

$f(\lambda)$ and the constraint functions $g_{1}(\lambda)$ and $g_{2}(\lambda)$ can be formulated as

$$
L\left(\lambda, \mu_{1}, \mu_{2}\right)=f(\lambda)+\mu_{1} \cdot g_{1}(\lambda)+\mu_{2} \cdot g_{2}(\lambda)
$$

Suppose the object function $f(\lambda)$ and the constraint functions $g_{1}(\lambda)$ and $g_{2}(\lambda)$ are continuously differentiable at a point $\lambda^{*}$. If $\lambda^{*}$ is a local minimum that satisfies some regular conditions (see below), then there exist constants $\mu_{1}$ and $\mu_{2}$ called KKT multipliers, such that

$$
\begin{aligned}
& \frac{\partial L\left(\lambda, \mu_{1}, \mu_{2}\right)}{\partial \lambda} \mid \lambda=\lambda^{*}=0 \\
& \mu_{1} \cdot g_{1}\left(\lambda^{*}\right)=0 \\
& \mu_{2} \cdot g_{2}\left(\lambda^{*}\right)=0 \\
& \mu_{1} \geq 0 \\
& \mu_{2} \geq 0
\end{aligned}
$$

Equation (23) can be rewritten as

$$
\begin{gathered}
\frac{\left(2 \sigma_{n}^{2}+\sigma_{s}^{2}\right) \cdot \lambda^{* 2}}{2 \sigma_{n}^{2}\left(\sigma_{n}^{2}+\sigma_{s}^{2}\right)}-\lambda^{*}-\frac{2 \cdot \sigma_{n}^{2}\left(\sigma_{n}^{2}+\sigma_{s}^{2}\right)}{\sigma_{s}^{2} N} \cdot \ln \frac{(1-\alpha) \cdot\left(\sigma_{n}^{2}+\sigma_{s}^{2}\right)}{\alpha \sigma_{n}^{2}}=0 \\
\mu_{1} \cdot\left(\lambda^{*}+\frac{1.28\left(\sigma_{n}^{2}+\sigma_{s}^{2}\right)}{\sqrt{N / 2}}-\sigma_{n}^{2}-\sigma_{s}^{2}\right)=0 \\
\mu_{2} \cdot\left(\lambda^{*}-\frac{1.28 \sigma_{n}^{2}}{\sqrt{N / 2}}-\sigma_{n}^{2}\right)=0
\end{gathered}
$$

$$
\mu_{1} \geq 0
$$

$$
\mu_{2} \geq 0
$$

By discussing all the possible cases of $\mu_{1}$ and $\mu_{2}$, a general solution for any $\alpha$ and $N$ can be obtained as follow

Case 1: $\mu_{1}=0, \mu_{2}=0$
The optimization equation (24) will be equivalent to the original one (17) without considering the constraint region. The solution is

$$
\lambda^{*}=\frac{1+\sqrt{1+\frac{4\left(2 \sigma_{n}^{2}+\sigma_{s}^{2}\right)}{N \sigma_{s}^{2}} \cdot \ln \left(\frac{(1-\alpha)\left(\sigma_{s}^{2}+\sigma_{n}^{2}\right)}{\alpha \sigma_{n}^{2}}\right)}}{\left(2 \sigma_{n}^{2}+\sigma_{s}^{2}\right) / \sigma_{n}^{2}\left(\sigma_{n}^{2}+\sigma_{s}^{2}\right)}
$$

where $\lambda^{*}$ should satisfy the following two conditions (30) and (31)

$$
\begin{gathered}
g_{1}\left(\lambda^{*}\right)=\lambda^{*}+\frac{1.28\left(\sigma_{n}^{2}+\sigma_{s}^{2}\right)}{\sqrt{N / 2}}-\sigma_{n}^{2}-\sigma_{s}^{2} \leq 0 \\
g_{2}\left(\lambda^{*}\right)=-\lambda^{*}+\frac{1.28 \sigma_{n}^{2}}{\sqrt{N / 2}}+\sigma_{n}^{2} \leq 0
\end{gathered}
$$

Equations (30) and (31) can be further derived by substituting $\lambda^{*}$ into $g_{1}(\lambda)$ and $g_{2}(\lambda)$ as

$$
\begin{aligned}
g_{1}\left(\lambda^{*}\right)= & \frac{1+\sqrt{1+\frac{4\left(2 \sigma_{n}^{2}+\sigma_{s}^{2}\right)}{N \sigma_{s}^{2}} \cdot \ln \left(\frac{(1-\alpha)\left(\sigma_{s}^{2}+\sigma_{n}^{2}\right)}{\alpha \sigma_{n}^{2}}\right)}}{\left(2 \sigma_{n}^{2}+\sigma_{s}^{2}\right) / \sigma_{n}^{2}\left(\sigma_{n}^{2}+\sigma_{s}^{2}\right)} \\
& +\frac{1.28\left(\sigma_{n}^{2}+\sigma_{s}^{2}\right)}{\sqrt{N / 2}}-\sigma_{n}^{2}-\sigma_{s}^{2} \\
g_{2}\left(\lambda^{*}\right)= & -\frac{1+\sqrt{1+\frac{4\left(2 \sigma_{n}^{2}+\sigma_{s}^{2}\right)}{N \sigma_{s}^{2}} \cdot \ln \left(\frac{(1-\alpha)\left(\sigma_{s}^{2}+\sigma_{n}^{2}\right)}{\alpha \sigma_{n}^{2}}\right)}}{\left(2 \sigma_{n}^{2}+\sigma_{s}^{2}\right) / \sigma_{n}^{2}\left(\sigma_{n}^{2}+\sigma_{s}^{2}\right)} \\
& +\frac{1.28 \sigma_{n}^{2}}{\sqrt{N / 2}}+\sigma_{n}^{2}
\end{aligned}
$$

For $\mathrm{N}=16384, \alpha=50 \%$, it can be found $g_{1}(\lambda) \leq 0$, when $S N R=\frac{\sigma_{s}^{2}}{\sigma_{n}^{2}} \geq-15.385 \mathrm{~dB} . g_{2}(\lambda) \leq 0$, when $S N R=\frac{\sigma_{s}^{2}}{\sigma_{n}^{2}} \geq$ $-15.461 \mathrm{~dB}$. Therefore, when $S N R=\frac{\sigma_{s}^{2}}{\sigma_{n}^{2}} \geq-15.385 \mathrm{~dB}$, the constraint region can be satisfied for $\mu_{1} \stackrel{n}{=} 0, \mu_{2}=0$.

Case 2: $\mu_{1}>0, \mu_{2}=0$

According to (24),

$$
\lambda^{*}=\frac{1+\sqrt{1+\frac{4\left(2 \sigma_{n}^{2}+\sigma_{s}^{2}\right)}{N \sigma_{s}^{2}} \cdot\left(\ln \left(\frac{(1-\alpha)\left(\sigma_{s}^{2}+\sigma_{n}^{2}\right)}{\alpha \sigma_{n}^{2}}\right)+\mu_{1}\right)}}{\left(2 \sigma_{n}^{2}+\sigma_{s}^{2}\right) / \sigma_{n}^{2}\left(\sigma_{n}^{2}+\sigma_{s}^{2}\right)}
$$

For $\mu_{1}>0$, the solution of (25) is

$$
\lambda_{1}=\sigma_{n}^{2}+\sigma_{s}^{2}-\frac{1.28\left(\sigma_{n}^{2}+\sigma_{s}^{2}\right)}{\sqrt{N / 2}}
$$

Therefore, $\lambda_{1}$ should be equal to $\lambda^{*}$ and both of them should make $g_{2}(\lambda) \leq 0$. However, the solution doesn't exist after computation. In other words, the case of $\mu_{1}>0, \mu_{2}=0$ doesn't exist as well.

Case 3: $\mu_{1}=0, \mu_{2}>0$

According to (24),

$$
\lambda^{*}=\frac{1+\sqrt{1+\frac{4\left(2 \sigma_{n}^{2}+\sigma_{s}^{2}\right)}{N \sigma_{s}^{2}} \cdot\left(\ln \left(\frac{(1-\alpha)\left(\sigma_{s}^{2}+\sigma_{n}^{2}\right)}{\alpha \sigma_{n}^{2}}\right)-\mu_{2}\right)}}{\left(2 \sigma_{n}^{2}+\sigma_{s}^{2}\right) / \sigma_{n}^{2}\left(\sigma_{n}^{2}+\sigma_{s}^{2}\right)}
$$

For $\mu_{2}>0$, the solution of (26) is

$$
\lambda_{2}=\frac{1.28 \sigma_{n}^{2}}{\sqrt{N / 2}}+\sigma_{n}^{2}
$$




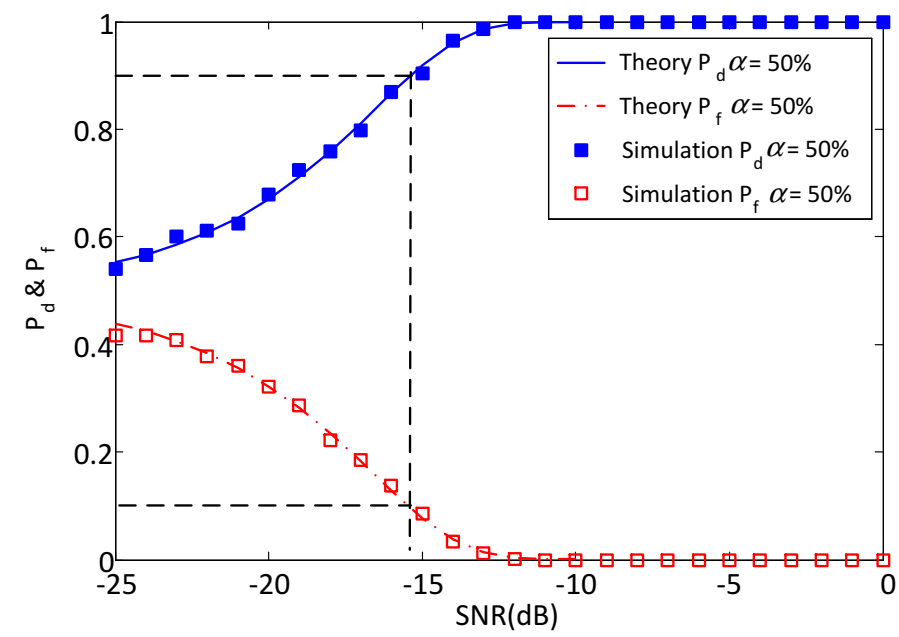

Fig. 3: $P_{d}$ and $P_{f}$ versus SNR for simulation and theory line with $\alpha=50 \%$

Therefore, $\lambda_{2}$ should be equal to $\lambda^{*}$ and both of them should make $g_{1}(\lambda) \leq 0$. For $\mathrm{N}=16384, \alpha=50 \%$, it can be found $g_{1}(\lambda) \leq 0$, when $S N R=\frac{\sigma_{s}^{2}}{\sigma_{n}^{2}} \geq-15.4212 \mathrm{~dB}$. The corresponding threshold $\lambda_{2}=1.0141$ for $\lambda_{n}{ }^{2}=1$. It can be proved there exist $\lambda^{*}=\lambda_{2}=1.0141$ to make the condition (24) satisfied.

Case4: $\mu_{1}>0, \mu_{2}>0$

For $\mu_{1}>0, \mu_{2}>0$, the solutions of (25) and (26) are

$$
\begin{gathered}
\lambda_{1}=\sigma_{n}^{2}+\sigma_{s}^{2}-\frac{1.28\left(\sigma_{n}^{2}+\sigma_{s}^{2}\right)}{\sqrt{N / 2}} \\
\lambda_{2}=\frac{1.28 \sigma_{n}^{2}}{\sqrt{N / 2}}+\sigma_{n}^{2}
\end{gathered}
$$

Therefore, $\lambda^{*}=\lambda_{1}=\lambda_{2}$, which can be thought as a special case of Case 3. It can be easily proved, all the conditions can be satisfied. All in all, considering all the four cases, we can conclude $\lambda^{*}=1.0143$ is the minimum threshold that could satisfy all the conditions in (23) with $\alpha=50 \%, \mathrm{~N}=16384$.

In order to verify our derivation, an example of $\alpha=50 \%$, $\mathrm{N}=16384$ is substituted into the obtained general solution. It can be proved that there exists such minimum $\lambda^{*}=1.0143$, $\mu_{1}$ and $\mu_{2}$ such that all the conditions (23) are satisfied when $\alpha=50 \%, \mathrm{~N}=16384$. Furthermore, the corresponding SNR is -15.3850 if the minimum $\lambda^{*}=1.0143$. Therefore, it can be found in Fig. 3 that the simulation curves are well matched to the theory curves, which proves our theoretical analysis.

The analytical study is then extended to a range of different number of samples $N(\mathrm{~N}=8192,16384,65536)$ as shown in Fig. 4. It can be found that the satisfied SNR value, considering the constraint region condition, decreases as the increase of the number of samples $N$. A similar result can be found in Fig. 5, the increased samples $N$ improves the performance for both $P_{d}$ and $P_{f}$. A higher $P_{d}$ or lower $P_{f}$ value can be obtained with the increase of the number of samples $N$ or SNR. Therefore, the more number of samples $N$ there are, the easier the constraint region condition can be satisfied. This is echoed by the results obtained by other literature [34], [35].

The effect of spectrum utilization $\alpha$ on the value of satisfied SNR considering the constraint region condition is analyzed

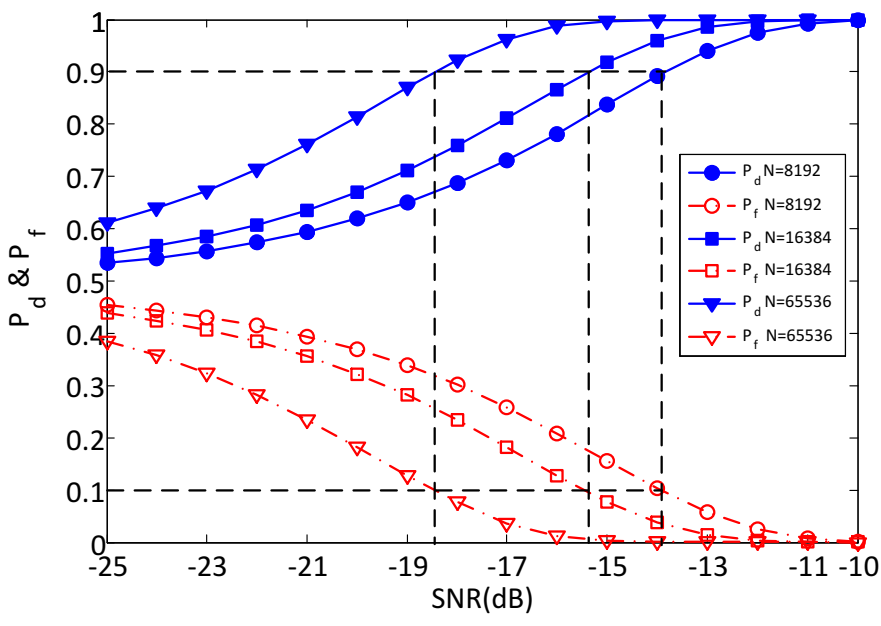

Fig. 4: $P_{d}$ and $P_{f}$ versus SNR for different number of samples $N$

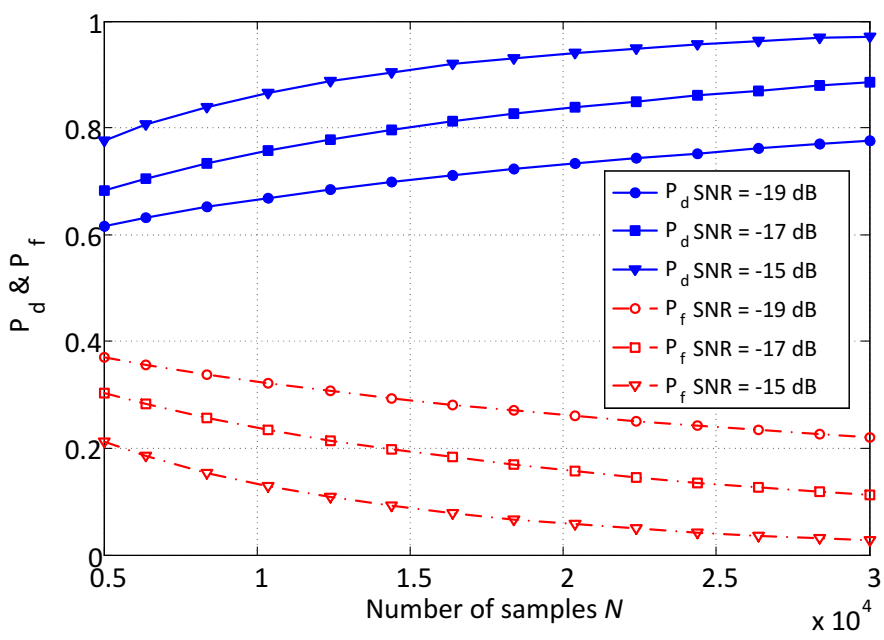

Fig. 5: $P_{d}$ and $P_{f}$ versus number of samples for different SNR

in Fig. 6 as well. It is found that the satisfied SNR values for both $10 \%$ and $90 \%$ cases show a symmetry property. In addition, it can be seen that the satisfied SNR values for both $10 \%$ and $90 \%$ cases are a bit higher than that of the $50 \%$ case. This is mainly due to the poor performance generated by both $10 \%$ and $90 \%$ cases in terms of either $P_{d}$ or $P_{f}$. The results can be useful in CR network design and deployment, such as anticipating the minimum SNR level that can be sensed under certain PU spectrum utilization or samples. The results also show that the sensing performance is degraded by either bursty activity patterns of PUs or SUs in practice.

\section{ImPlementation With the Welch AND WaVELET BASED ENERGy DETECTORS}

\section{A. Experiment Setup}

In this part, an LTE-A system with cognitive radio enhancement is being developed from [25] and the well-known LTE downlink OFDM signal is chosen for sensing. The simulation performance of the fixed threshold setting and previously proposed adaptive threshold setting algorithms are observed through implementations with both Welch and wavelet based energy detectors. In simulations, the number of samples is set 


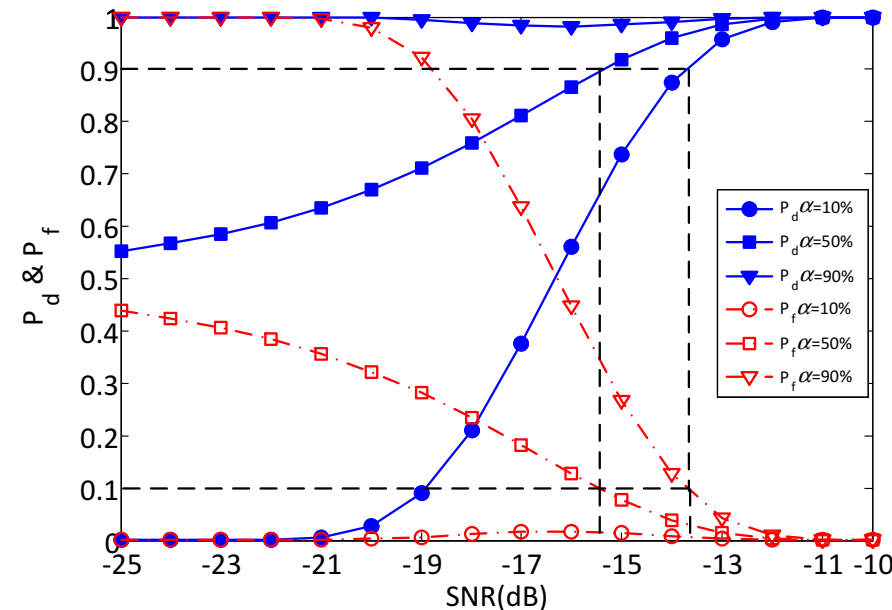

Fig. 6: $P_{d}$ and $P_{f}$ versus SNR for different PUs' spectrum utilizations $\alpha$

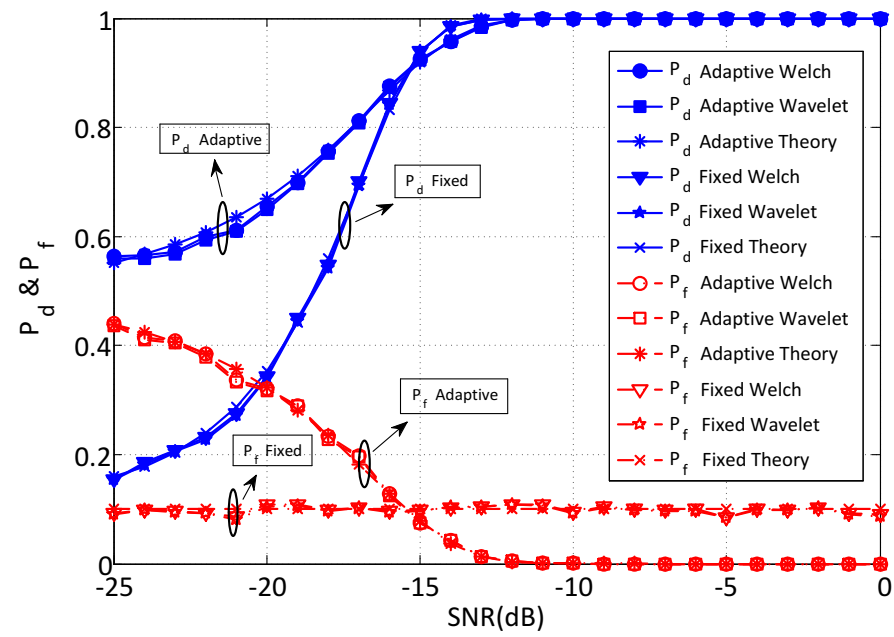

Fig. 7: $P_{d}$ and $P_{f}$ versus SNR for fixed/adaptive Welch and wavelet algorithms

to $\mathrm{N}=16384$, which is the same as that in the theoretical analysis in Section IV and V. The desired probability of false alarm for the fixed threshold is set as $P_{f}=0.1$. The initial spectrum utilization of the primary users is set as the worse case, $\alpha=50 \%$. The Welch and wavelet algorithms employed in this session are based on the sensing algorithms used in [16] and [36], respectively.

\section{B. Results and Analysis}

Monte-carlo simulations results shown in Fig. 7 show that both Welch and wavelet algorithms' results are very close to each other and match the theoretical one. Therefore, the correctness and reliability of our simulation platform is verified. The comparison of detection performance $P_{d}$ between the Welch and wavelet algorithms by implementing the proposed adaptive threshold setting algorithm is shown in Fig. 7. It is found that both Welch and wavelet algorithms with the proposed threshold setting can achieve higher detection probabilities than the conventional fixed one in the lower SNR region.

It can also be found in Fig. 7 that the false alarm probability $P_{f}$ of the proposed algorithm drops quickly for both Welch

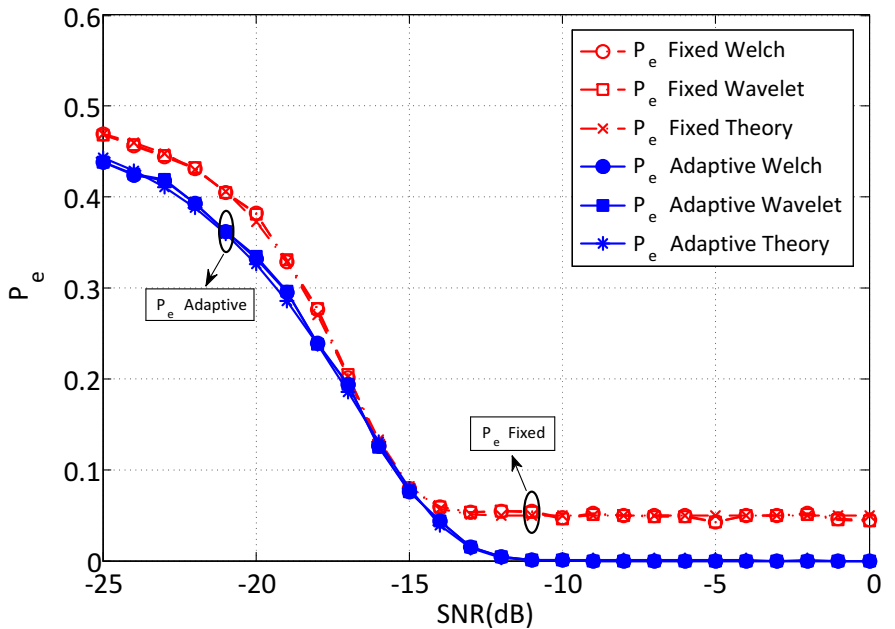

Fig. 8: $P_{e}$ versus SNR for fixed/adaptive Welch and wavelet algorithms

and wavelet algorithms compared to the conventional fixed one when the SNR is greater than $-15 \mathrm{~dB}$. This is because the proposed adaptive threshold is an increasing function with the increase of SNR values for $\alpha=50 \%$, which has been observed in Fig. 1 in [16]. The adaptive threshold is equal to the fixed one at SNR $=-15 \mathrm{~dB}$ and further increases for the $50 \%$ spectrum utilization. Therefore, the proposed adaptive threshold performs better in terms of the detection probability $P_{d}$ when the SNR value is lower than $-15 \mathrm{~dB}$ and a better false alarm performance $P_{f}$ when the SNR is greater than $-15 \mathrm{~dB}$. For the convectional fixed threshold method, $P_{f}$ remains the same under any SNR environments since $P_{f}$ is considered for the case of no signal transmission and such is independent of SNR. However, the overall performance of the adaptive threshold outperforms the fixed threshold in terms of the error decision probability $P_{e}$ as shown Fig. 8. In Fig. 8, both Welch and wavelet algorithms' error decision probability $P_{e}$ match well with that of the theory. Moreover, the proposed adaptive algorithms could always achieve a lower error decision probability $P_{e}$ compared to that of the conventional fixed one.

By implementing the wavelet de-noising method, the detection performance $P_{d}$ for both fixed and proposed adaptive threshold setting algorithm with the corresponding false alarm performance $P_{f}$ is shown in Fig. 9. With the wavelet denoising function, both fixed and adaptive wavelet methods can achieve a higher detection probability $P_{d}$ in comparison with the original ones without de-noising methods. Meanwhile, the wavelet de-noising method increases the false alarm probability $P_{f}$ a little for both fixed and adaptive wavelet methods. However, in comparison with the fixed wavelet de-noising method, the adaptive wavelet de-noising method's false alarm probability $P_{f}$ drops quickly when SNR is greater than $-15 \mathrm{~dB}$ and almost merges with the one without de-noising method.

The corresponding error decision probability $P_{e}$ is shown in Fig. 10. It is found that both adaptive wavelet de-noising and without de-noising algorithms' error decision probability $P_{e}$ can always keep a relatively low value compared to the fixed one. This means a more reliable and robust overall sensing result for both PUs and SUs. In summary, the proposed adaptive threshold methods outperform the fixed ones for the 


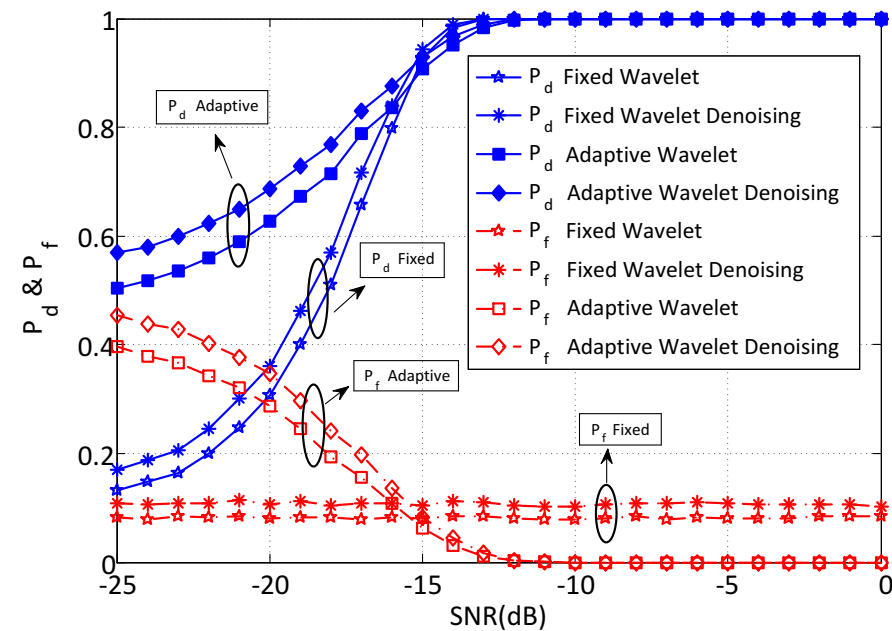

Fig. 9: $P_{d}$ and $P_{f}$ versus SNR for wavelet algorithms with/without de-noising

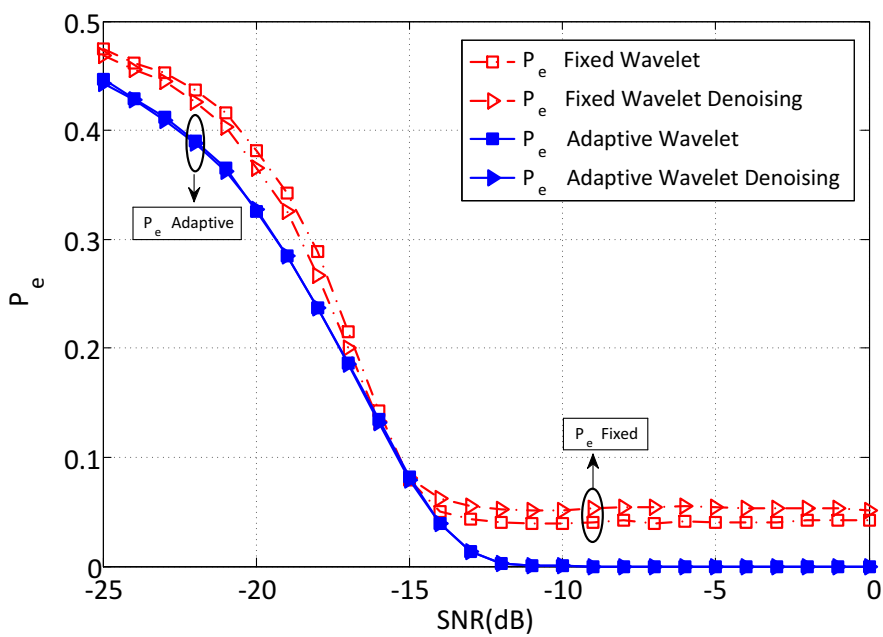

Fig. 10: $P_{e}$ versus SNR for fixed/adaptive wavelet algorithms with/without de-noising

cases of both de-noising and without de-noising. The denoising methods can improve the wavelet method's sensing performance in comparison with the one without de-noising.

In addition, a comparison of the computation complexity in terms of the real multiplication is made between the Welch and wavelet based energy detection algorithms as follows [37]. The number of samples is set to $N$.

$$
\begin{aligned}
& \text { Welch: } \\
& =2 N \log _{2} N \\
& \text { db5 FIR wavelet filtering schemes for DWPT: } \\
& 10 \text { coeff. } \times\left(2 N+\ldots+2^{\left(\log _{2} N\right)} N / 2^{\left(\log _{2} N-1\right)}\right) \\
& =10 \times\left(2 N \log _{2} N\right)
\end{aligned}
$$

where coeff. represents the coefficients. db5 is the wavelet basis used in our simulation. It can be found that the Welch algorithm has a lower computational complexity, which makes the spectrum sensing faster compared to the wavelet one. Although the wavelet algorithm results in a relatively high computation complexity, the wavelet de-noising method can be implemented to make a further sensing performance improvement as shown Fig. 9

Fig. 11 shows the performance of the proposed adaptive wavelet algorithm implemented with the de-noising method

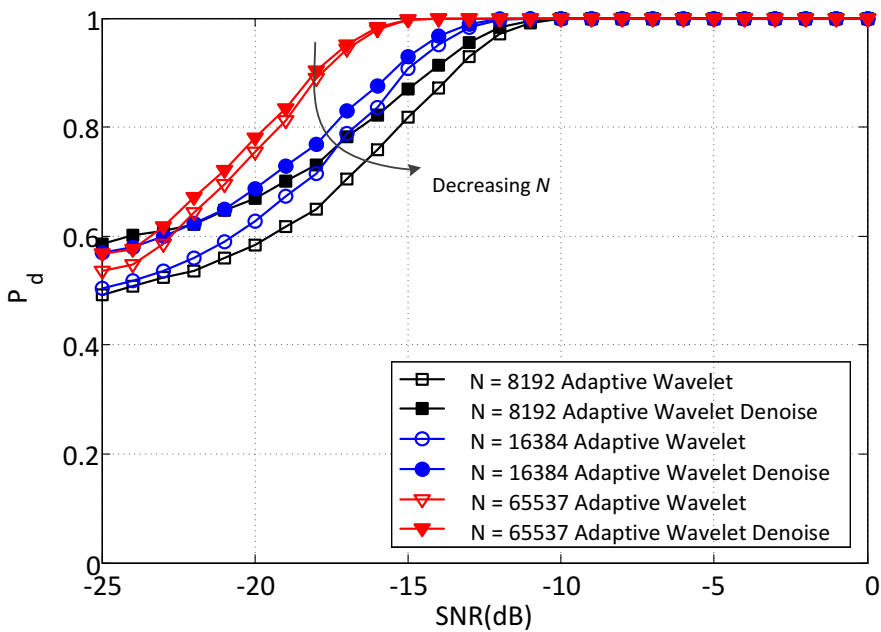

Fig. 11: $P_{d}$ versus SNR with different samples $\mathrm{N}$ for wavelet algorithms with/without de-noising

at different numbers of samples, especially when the number of samples $N$ is not very large. In reality, the number of samples $N$ at the level of thousands is always preferred due to the limitation of equipment. As shown in Fig. 11, by adopting the wavelet de-noising method, a bigger improvement can be obtained as the number of samples $N$ decreases.

\section{CONCLUSION}

Previous research has usually been limited to a fixed PUs' spectrum utilization and lacks consideration of the constraint region requirement set by the IEEE 802.22 standard. In this paper, both conventional fixed threshold setting algorithm and previously developped adaptive threshold setting algorithm have been modified by considering the constraint region requirement. The closed-form expression between satisfied SNR value and number of samples have been derived for the conventional fixed threshold setting algorithm. Mathematical expression between PU's spectrum utilization ratio $\alpha$ and the proposed adaptive threshold has been derived as well considering the constraint region requirement. Furthermore, both threshold setting algorithms have been verified by MonteCarlo simulations with the constraint region requirement. The numerical analysis demonstrated the significant impacts of PUs' spectrum utilization on the overall system sensing reliability. Both analytical and numerical results have shown that a lower error decision probability can be obtained for the proposed adaptive threshold setting algorithm in comparison with the conventional fixed one. Overall, the analytical study on the PUs' spectrum utilizations and the constraint region can provide practical guidance when designing a cognitive LTE system for the future $5 \mathrm{G}$ wireless communications.

\section{ACKNOWLEDGEMENT}

This work is supported by the EPSRC TV White Space Testbed with Robust Spectrum Sensing Algorithms Project with Grant No. EP/L024241/1 in the UK, and the State Major Science and Technology Special Project with Grant No. 2015ZX03002004 in China. 


\section{REFERENCES}

[1] J. Mitola, III and G. Q. Maguire., Jr., "Cognitive radio: making software radios more personal," IEEE Pers. Commun., vol. 6, no. 4, pp. 13-18, Aug. 1999.

[2] S. Haykin, "Cognitive radio: brain-empowered wireless communications," IEEE J. Sel. Areas Commun., vol. 23, no. 2, pp. 201-220, Feb. 2005.

[3] T. Kaiser, H. Cao, W. Jiang, and F. Zheng, "Cognitive radio - a current snapshot and some thoughts on commercialization for future cellular systems," Journal of Signal Processing Systems, vol. 73, no. 3, pp. 217 225, Aug. 2013.

[4] H. Cao, W. Jiang, and T. Kaiser, "Parallel in-band signal detection with self-interference suppression for cognitive lte," Proc. IEEE Wireless Commun. Netw. Conf. (WCNC'14), pp. 64-69, Apr. 2014.

[5] M. Nekovee, "Impact of cognitive radio on future management of spectrum," 3rd International Conference on Cognitive Radio Oriented Wireless Networks and Communications (CrownCom), pp. 1-6, May. 2008.

[6] Z. Qin, Y. Gao, M. Plumbley, and C. Parini, "Wideband spectrum sensing on real-time signals at sub-nyquist sampling rates in single and cooperative multiple nodes," IEEE Trans. Signal Process., vol. PP, no. 99 , pp. 1-1, 2016.

[7] M. Tavana, A. Rahmati, V. Shah-Mansouri, and B. Maham, "Cooperative sensing with joint energy and correlation detection in cognitive radio networks," IEEE Commun Lett., vol. 21, no. 1, pp. 132-135, Jan 2017.

[8] O. Semiari, B. Maham, and C. Yuen, "On the effect of i/q imbalance on energy detection and a novel four-level hypothesis spectrum sensing," IEEE Trans. Veh. Tech., vol. 63, no. 8, pp. 4136-4141, Oct 2014

[9] "Ieee 802.22/d1.0 draft standard for wireless regional area networks part 22: Cognitive wireless ran medium access control (mac) and physical layer (phy) specifications: Policies and procedures for operation in the tv bands," Apr. 2008.

[10] P. Van Wesemael, S. Pollin, E. Lopez, and A. Dejonghe, "Performance evaluation of sensing solutions for lte and dvb-t," Proc. IEEE Symp. Dynamic Spectrum Access Networks (DySPAN ',11)., pp. 531-537, May. 2011.

[11] A. Makarfi and K. Hamdi, "Interference analysis of energy detection for spectrum sensing," IEEE Trans. Veh. Tech., vol. 62, no. 6, pp. 25702578, Jul. 2013.

[12] D. Cabric, A. Tkachenko, and R. W. Brodersen, "Experimental study of spectrum sensing based on energy detection and network cooperation," Proc. of First Int. Workshop on Technology and Policy for Accessing Spectrum (TAPAS), Aug. 2006.

[13] D. Martinez and A. Andrade, "Performance evaluation of welch's periodogram-based energy detection for spectrum sensing," IET Commun, vol. 7, no. 11, pp. 1117-1125, Jul. 2013.

[14] T. Yucek and H. Arslan, "A survey of spectrum sensing algorithms for cognitive radio applications," IEEE Comm. Surv. and Tut., vol. 11, no. 1, pp. 116-130, Jan. 2009.

[15] E. Axell, G. Leus, E. Larsson, and H. Poor, "Spectrum sensing for cognitive radio : State-of-the-art and recent advances," IEEE Signal Process. Mag., vol. 29, no. 3, pp. 101-116, May. 2012.

[16] N. Wang, Y. Gao, and X. Zhang, "Adaptive spectrum sensing algorithm under different primary user utilizations," IEEE Commun Lett., vol. 17, no. 9, pp. 1838-1841, Sep. 2013.

[17] Z. Qin, Y. Gao, and C. Parini, "Data-assisted low complexity compressive spectrum sensing on real-time signals under sub-nyquist rate," IEEE Trans. Wireless Commun., vol. 15, no. 2, pp. 1174-1185, Feb. 2016.

[18] N. Wang, Y. Gao, and B. Evans, "Database-augmented spectrum sensing algorithm for cognitive radio," Proc. IEEE Int. Conf. Commun. (ICC'15), accepted, Jun. 2015.

[19] Y. Hassan, M. El-Tarhuni, and K. Assaleh, "Comparison of linear and polynomial classifiers for co-operative cognitive radio networks," Proc. IEEE Int. Symp. PIMRC Workshops, pp. 797-802, Sep. 2010.

[20] J. Chen, A. Gibson, and J. Zafar, "Cyclostationary spectrum detection in cognitive radios," IET Seminar on Cognitive Radio and Software Defined Radios: Technologies and Techniques, pp. 1-5, Sep. 2008.

[21] US Federal Communications Commission (FCC), Evaluation of the Performance of Prototype TV-Band White Space Devices Phase II, OET Report: FCC/OET 08-TR-1005, Oct. 2008.

[22] UK Office of Communications (Ofcom), Staement on Cognitive Access to Interleaved Spectrum, Jul. 2009.

[23] Z. Ye, J. Grosspietsch, and G. Memik, "Spectrum sensing using cyclostationary spectrum density for cognitive radios," Proc. IEEE Workshop on Signal Process Systems, pp. 1-6, Oct. 2007.

[24] P. Cheraghi, Y. Ma, R. Tafazolli, and Z. Lu, "Cluster-based differential energy detection for spectrum sensing in multi-carrier systems," IEEE Trans. Signal Process., vol. 60, no. 12, pp. 6450-6464, Dec. 2012.
[25] N. Wang and Y. Gao, "Optimal threshold of welch periodogram for sensing ofdm signals at low snr levels," Proc. Eur. Wireless Conf. (EW'13)., pp. 1-5, Apr. 2013.

[26] T. Zhao and Y. Zhao, "A new cooperative detection technique with malicious user suppression," Proc. IEEE Int. Conf. on Commun (ICC '09)., pp. 1-5, Jun. 2009.

[27] Z. Ye, G. Memik, and J. Grosspietsch, "Energy detection using estimated noise variance for spectrum sensing in cognitive radio networks," Proc. IEEE Wireless Commun. Netw. Conf. (WCNC'08), pp. 711-716, Apr. 2008.

[28] Y.-E. Lin, K.-H. Liu, and H.-Y. Hsieh, "On using interference-aware spectrum sensing for dynamic spectrum access in cognitive radio networks," IEEE Trans. Mobile Computing., vol. 12, no. 3, pp. 461-474, Mar. 2013.

[29] E. Peh and Y.-C. Liang, "Optimization for cooperative sensing in cognitive radio networks," Proc. IEEE Wireless Commun. Netw. Conf. (WCNC'07), pp. 27-32, Mar. 2007.

[30] O. Noskoski, J. Bermudez, and S. de Almeida, "Region-based waveletpacket adaptive algorithm for identification of sparse impulse responses," IEEE Trans. Signal Process., vol. 61, no. 13, pp. 3321-3333, Jul. 2013.

[31] D. L. Donoho and J. M. Johnstone, "Ideal spatial adaptation by wavelet shrinkage," Biometrika, vol. 81, no. 3, pp. 425-455, Aug. 1994.

[32] L. Sendur and I. Selesnick, "Bivariate shrinkage functions for waveletbased denoising exploiting interscale dependency," IEEE Trans. Signal Process., vol. 50, no. 11, pp. 2744-2756, Nov. 2002.

[33] S. Boyd and L. Vandenberghe, Convex optimization. Cambridge: Cambridge University Press, Mar. 2004.

[34] C. Guo, X. Wu, C. Feng, and Z. Zeng., "Spectrum sensing for cognitive radios based on directional statistics of polarization vectors," IEEE $J$. Sel. Areas Commun., vol. 31, no. 3, pp. 379-393, Mar. 2013.

[35] E. Hassan, "Spectrum sensing and power efficiency trade-off optimisation in cognitive radio networks over fading channels," IET Commun, vol. 7, no. 3, pp. 198-205, Feb. 2013.

[36] Z. Qin, N. Wang, Y. Gao, and L. Cuthbert, "Adaptive threshold for energy detector based on discrete wavelet packet transform," Proc. IEEE Wireless Telecomm Symp (WTS'12), pp. 1-5, Apr. 2012.

[37] Y. Youn, H. Jeon, H. Jung, and H. Lee, "Discrete wavelet packet transform based energy detector for cognitive radios," Proc. IEEE 65th Veh. Technol. Conf. (VTC'07), pp. 2641-2645, Apr. 2007. 